\title{
Secretion of IL-16 through TNFR1 and calpain-caspase signaling contributes to MRSA pneumonia
}

\author{
DS Ahn ${ }^{1}$, D Parker ${ }^{1}$, PJ Planet ${ }^{1}$, PA Nieto ${ }^{2,3}$, SM Bueno $^{2,3}$ and A Prince ${ }^{1,4}$
}

Staphylococcus aureus is a major cause of severe pneumonia. Multiple mechanisms of proinflammatory signaling are activated to recruit immune cells into the airway in response to $S$. aureus. We found that interleukin-16 (IL-16), a T cell cytokine that binds $\mathrm{CD} 4$, is potently activated by $S$. aureus, specifically by protein $\mathrm{A}(\mathrm{SpA})$, and to a much greater extent than by Gram-negative pathogens or lipopolysaccharide. IL-16 production involved multiple signals including ligation of tumor necrosis factor receptor (TNFR) family members or epidermal growth factor receptor, both receptors for $\mathrm{SpA}$ and generation of $\mathrm{Ca}^{2+}$ fluxes to activate calpains and caspase-3. Although human airway epithelial cells, vascular endothelial cells, THP-1 and Jurkat Tcells released IL-16 in response to S. aureus in vitro, in a murine model of pneumonia, $\mathrm{CD} 4{ }^{+}$cells were the major source of IL-16 suggesting the involvement of an autocrine signaling pathway. The production of IL-16 contributed to lung damage as neutralization of IL-16 enhanced $S$. aureus clearance and resulted in diminished lung pathology in S. aureus pneumonia. Our results suggest that the ability of $S$. aureus to activate TNFR1 and $\mathrm{Ca}^{2+} / \mathrm{cal}_{\text {pain }}$ signaling contribute to $T$ cell activation and excessive inflammation in the setting of acute pneumonia.

\section{INTRODUCTION}

Staphylococcus aureus, particularly the epidemic USA300 methicillin-resistant $S$. aureus (MRSA), is a major cause of pneumonia. ${ }^{1}$ Common in health care-associated settings, MRSA is a frequent cause of ventilator-associated pneumonia ${ }^{2}$ and superinfection complicating influenza. ${ }^{3}$ Even though there are antimicrobials with good activity against MRSA, there remains significant morbidity and mortality associated with this pathogen. ${ }^{1,4}$ A substantial literature suggests that specific staphylococcal virulence factors are directly responsible for lung injury such as $\alpha$-hemolysin ${ }^{5}$ and Panton-Valentine leukocidin. ${ }^{6}$ However, much of the pathology associated with MRSA pneumonia can be attributed to the intensity of the host inflammatory response. S. aureus activates multiple redundant proinflammatory signaling cascades and murine models of S. aureus pneumonia lacking specific components of the innate immune signaling pathway such as type 1 interferon receptor, ${ }^{7}$ inflammasome protein $\mathrm{NLRP}^{8}$ and tumor necrosis factor receptor 1 (TNFR1) $)^{9}$ have improved bacterial clearance. $S$. aureus infection is accompanied by a significant TNF response and the abundant $S$. aureus surface component protein A (SpA), directly activates TNFR1 signaling. ${ }^{10}$

The recruitment of neutrophils and macrophages in response to chemokine and cytokine expression in the lung is a critical component of innate immune signaling in response to $S$. aureus. ${ }^{11,12}$ The contribution of T cells in the setting of acute staphylococcal infection is less well characterized, but is likely to be important ${ }^{13}$ as seen in Rag $2^{-/-}$mice, which exhibited resistance to $S$. aureus infection in a sepsis model. ${ }^{14}$ Not only are $\mathrm{T}$ cells essential to coordinate an adaptive immune response, they can be directly activated by $S$. aureus superantigens and are a major source of proinflammatory cytokines, ${ }^{15}$ such as TNF. Activated T cells contribute to pulmonary pathology in the setting of acute lung injury. CXCR3 is preferentially expressed in Thelper cells, ${ }^{16}$ and is increased in both infectious and noninfectious models of acute lung

\footnotetext{
${ }^{1}$ Departments of Pediatrics, Columbia University College of Physicians and Surgeons, New York, New York, USA. ${ }^{2}$ Millennium Institute on Immunology and Immunotherapy, Santiago, Chile. ${ }^{3}$ Departamento de Genética Molecular y Microbiología, Facultad de Ciencias Biológicas, Pontificia Universidad Católica de Chile, Santiago, Chile and ${ }^{4}$ Department of Pharmacology, Columbia University College of Physicians and Surgeon, New York, New York, USA. Correspondence: A Prince (asp7@columbia.edu) 
injury. ${ }^{11,12}$ Overexpression of the T cell chemokine CXCL10, a CXCR3 ligand, induced airway inflammation ${ }^{16}$ and infected CXCL10 knockout mice had decreased lung pathology. ${ }^{11}$ In acute bacterial infection, multiple $\mathrm{T}$ cell chemokines and cytokines are produced such as IL- $12{ }^{17}$ and IL-16, ${ }^{18}$ which serve to recruit $\mathrm{T}$ cells and stimulate the expression of surface receptors that mediate their proliferation and cytokine production.

IL-16 is a multifunctional cytokine with a single PDZ domain initially characterized as a product of human peripheral blood mononuclear cells and described as a lymphocyte chemoattractant ${ }^{19}$ (previously named lymphocyte chemoattractant factor). Transcribed as pro-IL-16, its production is regulated both at the level of transcription and caspase-3-dependent processing in various cell types. ${ }^{18}$ IL-16 induces chemotaxis of $\mathrm{CD}^{+}$cells such as lymphocytes, eosinophils, and dendritic cells by ligating CD4 directly at a site distinct from other ligands. ${ }^{18,19}$ Among its multiple functions, IL-16 is a T cell chemoattractant involved in $\mathrm{T}$ helper cell inflammatory responses and the regulation of both $\mathrm{T}$ cell growth, and responsiveness to regulatory cytokines. ${ }^{20}$ Chemotaxis of T cells induced by the supernatant of TNF-stimulated epithelial cells is inhibited by anti-IL-16 antibody, ${ }^{21}$ indicating a close association between IL-16 and TNF signaling. The processing of pro-IL-16 to its active form is mediated by caspase- $3^{22}$ via caspase- 8 . These caspases are activated following ligation of TNFR $1,{ }^{23}$ the target of SpA. ${ }^{10}$ In inflammatory conditions characterized by excessive TNF signaling, such as inflammatory bowel disease, IL-16 contributes significantly to pathology, ${ }^{24}$ thus we postulate that in the setting of robust TNF signaling, as occurs during S. aureus pneumonia, IL-16 similarly contributes to pathology.

In the experiments detailed herein, we describe the participation of IL-16 in S. aureus pneumonia and suggest that the unique ability of this organism to directly activate TNFR1, as well as the $\mathrm{Ca}^{2+} /$ calpain/caspase cascade, results in the release of this $\mathrm{T}$ cell cytokine.

\section{RESULTS}

\section{S. aureus induces IL-16 in the murine lung}

Significantly higher levels of IL-16 were recovered from the bronchoalveolar lavage fluid (BALF) of wild-type (WT) mice infected with $10^{7}$ colony-forming units of USA300 MRSA ( $P=0.0001$ compared with phosphate-buffered saline) or $S$. aureus 502A, a representative methicillin-sensitive strain. Lower levels were retrieved from mice infected with $10^{7}$ colonyforming unit of Gram-negative organisms such as Klebsiella pneumoniae ST258 and Pseudomonas aeruginosa PAK $(P<0.05$ compared with MRSA) (Figure 1a). To determine if human cells similarly had differential IL-16 responses to $S$. aureus vs. Gram-negative stimuli the human monocytic cells, THP-1s were stimulated with the same pathogens. IL-16 from THP-1s was 18-fold higher than the media control after incubation with MRSA $(P=0.0001)$ and 20 -fold after infection with $S$. aureus 502A. Less IL-16 was measured in response to infection with $K$. pneumoniae ST258 or PAK $(P<0.05$ compared with MRSA $)$ (Figure 1b).

\section{IL-16 secretion is SpA dependent in specific cell types}

The major $S$. aureus surface component SpA activates TNFR1 signaling, ${ }^{10}$ a receptor expected to activate IL-16 processing. The ability of the WT MRSA, a spa null mutant, and purified SpA to stimulate IL-16 in several cell types was compared (Figure 2). Induction of IL-16 by the spa null mutant was significantly decreased as compared with MRSA in human bronchial epithelial cells $(16 \mathrm{HBEs})(P=0.028)$ (Figure 2a), human umbilical vein endothelial cells (HUVECs) $(P=0.002)$ (Figure 2b) and T cells (Jurkats) $(P=0.06)$ (Figure 2c); WT MRSA and the spa null mutant stimulated similar amounts of IL-16 in monocytes (THP-1s) (Figure 2d). SpA alone induced IL-16 release from 16HBEs, HUVECs, Jurkats, and THP-1s (all $P<0.05$ as compared with media alone). Of note, lipopolysaccharide and TNF failed to stimulate IL-16 secretion in any of the cell types tested, with the exception of TNF-stimulated Jurkats $(P<0.05$ as compared with media).

\section{TNFR1 participates in IL-16 secretion}

TNFR1 ligation activates caspase 3 signaling, ${ }^{23}$ important in the processing of pro-IL- 16 to its active form ${ }^{22}$ and is stimulated by the IgG binding domain of SpA. ${ }^{10}$ To establish the importance of TNFR1 mediated signaling in the induction of IL-16 production, we compared generation of IL-16 in WT and Tnfr $1^{-/-}$mice (Figure 3). There was significantly less IL-16 in the BALF of infected Tnfr1 $1^{-/}$mice compared with WT controls $(P<0.05)$ (Figure 3a). Protein content in the BALF was slightly lower in the knockout mice (Figure $3 \mathbf{b}$ ) with similar levels of KC/CXCL1 (Figure 3c). Histopathology of Tnfr $1^{-1-}$ lungs infected with MRSA suggested less destruction of the normal architecture than WT mouse lung (Figure 3d), consistent with previous reports. ${ }^{10}$ Numbers of bacteria recovered in BALF were similar (Figure 3e) and recruited innate immune cells into BALF and lung were not significantly different in the WT and Tnfr1 ${ }^{-1-}$ mice (Figure 3f-h). Participation of TNFR1 in IL-16 processing is evident, but incomplete attenuation of IL-16 production in Tnfr $1^{-/-}$mice suggests that other pathways are involved.

\section{Multiple receptors participate in MRSA induced IL-16 secretion}

The direct association of SpA and IL-16 shown in stromal and $\mathrm{T}$ cells, indicates that additional SpA receptors may be involved in the generation of IL-16. Accordingly, we tested the participation of epidermal growth factor receptor (EGFR), a receptor for the IgG binding domain of $\mathrm{SpA}$ and directly activated in S. aureus infection. ${ }^{25}$ In the presence of blocking antibody to EGFR, MRSA induction of IL-16 secretion in THP1 s was significantly diminished $(P=0.023)$ as compared with a relevant IgG control (Figure 4a). Activation of Fas ligand (CD95), another member of the TNFR superfamily, ${ }^{23}$ also leads to pro-caspase 8 cleavage, ${ }^{26}$ which in turn activates caspase 3 from its proform, by $\mathrm{Ca}^{2+}$ influx and calpain activation. ${ }^{27}$ 

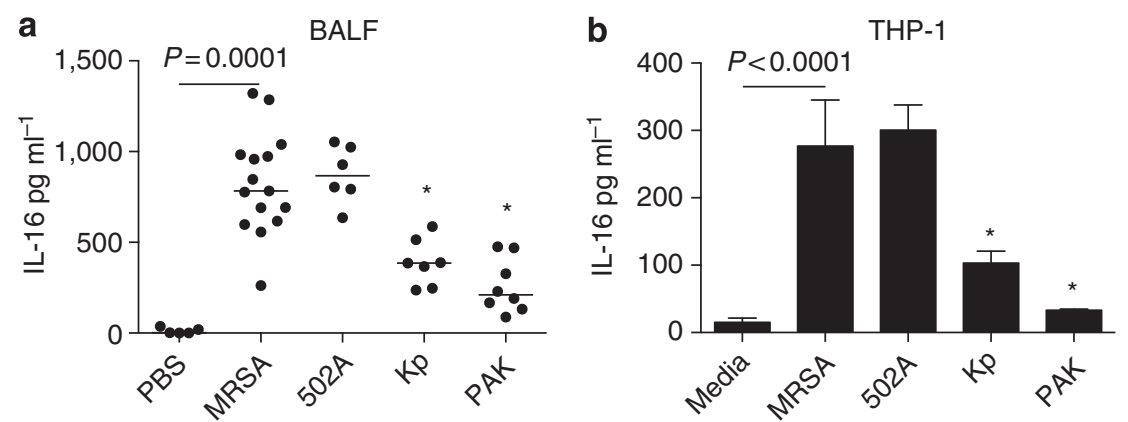

Figure 1 Staphylococcus aureus (S. aureus) induces interleukin-16 (IL-16) production in vivo and in vitro. (a) C57BL/6 J WT mice inoculated with $10^{7}$ colony-forming units of methicillin-resistant $S$. aureus USA300 (MRSA), S. aureus 502A, K. pneumoniae ST258 (Kp) or $P$. aeruginosa strain (PAK) or phosphate-buffered saline (PBS). Bronchoalveolar lavage fluid (BALF) was obtained $24 \mathrm{~h}$ after infection and quantitated by ELISA. Each dot represents an individual mouse; lines represent median values; data was compiled from two independent experiments. ${ }^{\star} P<0.05$ as compared with MRSA, ANOVA, post hoc Dunnett's test. Lines represent median values. (b) THP-1s were stimulated with MRSA, S. aureus 502A, Kp or PAK (MOI 100) ${ }^{\star} P<0.05$ compared with media, ANOVA, post hoc Dunnett's test. Graph shown is representative of two independent experiments.
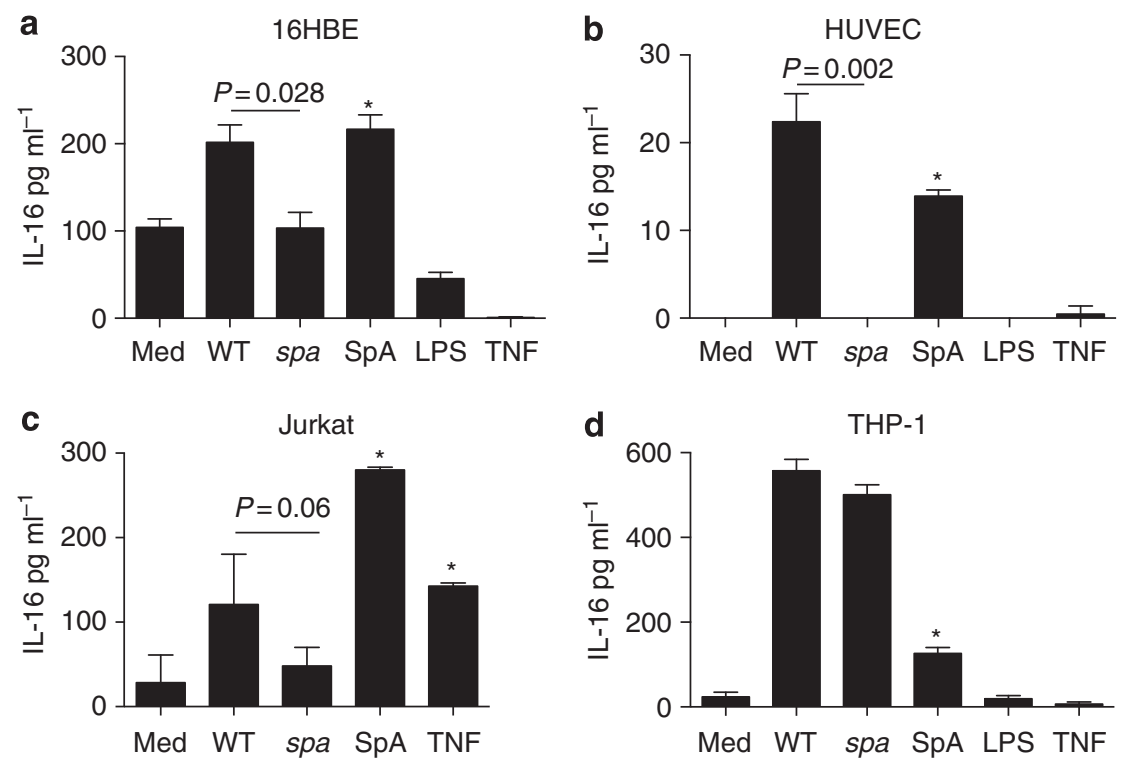

Figure 2 Interleukin-16 (IL-16) secretion is SpA specific in multiple cell types. (a) human bronchial epithelial cells (16HBEs), (b) human umbilical vein endothelial cells (HUVECs), (c) Jurkats and (d) THP-1s were infected with wild type (WT) methicillin-resistant S. aureus (MRSA) or spa mutant (MOI 100, $4 \mathrm{~h}$ for $16 \mathrm{HBEs}, 2 \mathrm{~h}$ for HUVECs, Jurkats and THP-1s). SpA, lipopolysaccharide (LPS), and TNF were added at a concentration of $0.5 \mu \mathrm{m}, 10 \mu \mathrm{g} / \mathrm{ml}$, and $100 \mu \mathrm{g} / \mathrm{ml}$, respectively. IL-16 in culture supernatants was quantified by ELISA ( ${ }^{\star} P<0.05$ compared with media, ANOVA, post hoc Dunnett's test). Graphs represent data from at least two independent experiments.

Pretreatment of THP-1s with anti-Fas ligand antibody before infection with MRSA also diminished the release of IL-16 $(P=0.0007)$ (Figure $4 \mathbf{b}) . \mathrm{As} \mathrm{Ca}^{2+}$ fluxes are generated by tolllike receptor 2 (TLR2) signaling, ${ }^{28}$ induced by $S$. aureus, ${ }^{29}$ we monitored IL-16 production by THP-1s pre-treated with antiTLR2 and found no diminution of IL-16 levels (Figure 4c). In addition, incubation of THP-1s with the TLR2 agonist $\mathrm{Pam}_{3}$ Cys-Ser-Lys $_{4}$ did not induce IL-16 production, indicating that TLR2 by itself is not responsible for IL-16 production. Thus, multiple TNFR effectors, as well as EGFR, participate in IL-16 secretion.

\section{Calcium, calpains, and caspases are involved in IL-16} processing

Our results suggest that multiple ligands that activate caspase- 8 and caspase- 3 could participate in the induction of pro-IL-16 processing. ${ }^{22}$ Given the participation of the $\mathrm{Ca}^{2+}$-dependent calpains in caspase activation, we examined the requirement of $\mathrm{Ca}^{2+}$ fluxes in the induction of IL-16. MRSA induced $\mathrm{Ca}^{2+}$ fluxes in Fluo-4/acetoxymethyl ester fluorescent dyes-loaded THP-1s within 10 min of exposure to MRSA with attenuation by pretreatment with 1,2 bis(2-aminophenoxy)ethaneN,N,N9,N9-tetra-acetic acid (BAPTA) (Figure $\mathbf{5 a}$ and 


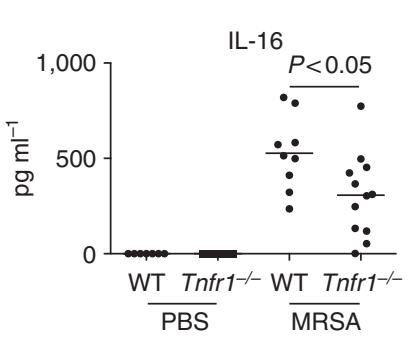

b

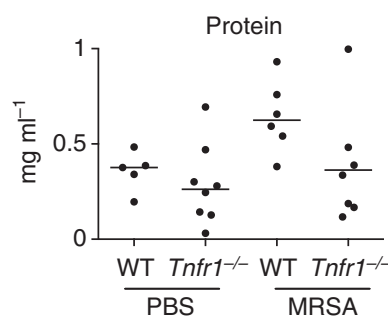

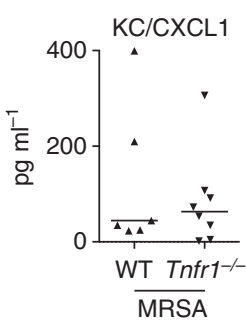

d

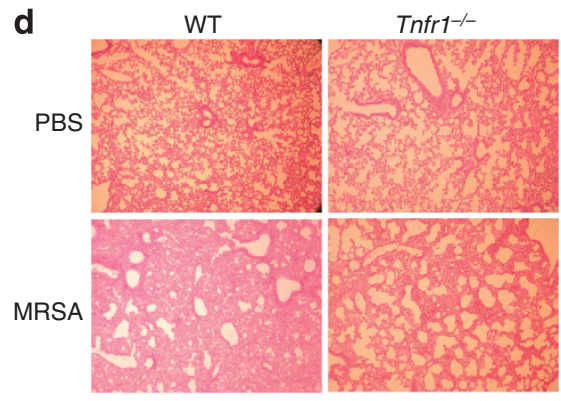

e

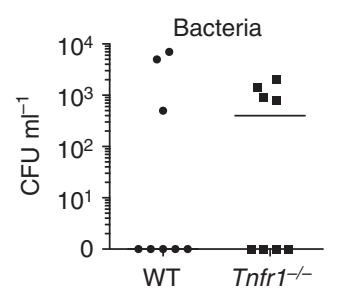

f

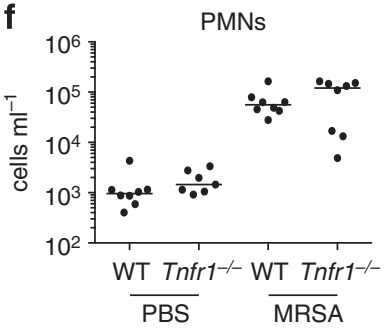

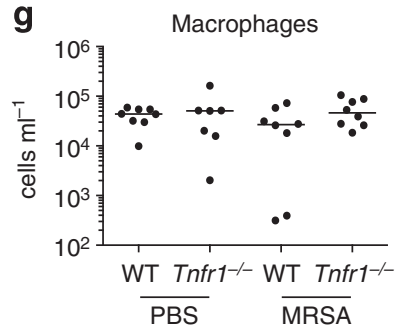

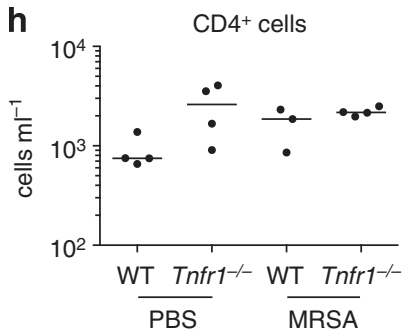

Figure 3 Tumor necrosis factor receptor 1 (TNFR1) is involved in interleukin-16 (IL-16) secretion. Age- and sex-matched cohorts of C57BL/6 J wild type (WT) and Tnfr $1^{-1-}$ mice were inoculated with $10^{7}$ colony-forming units (CFU) of methicillin-resistant S. aureus USA300 (MRSA) or phosphate-buffered saline (PBS) for $24 \mathrm{~h}$. Bronchoalveolar lavage fluid (BALF) was quantitated for (a) IL-16 by ELISA, (b) total protein (c) and KC/CXCL1 by ELISA. (d) hematoxylin and eosin stain sections of fixed-whole lungs. Magnification $\times 100$. (e) Bacterial CFUs were counted in BALF. (f) Polymorphonuclear neutrophils (PMNs) $\left(\mathrm{Ly}_{6 \mathrm{G}}{ }^{+} / \mathrm{MHCII}^{-}\right)$and $(\mathbf{g})$ Macrophages $\left(\mathrm{CD} 11 \mathrm{c}^{+} / \mathrm{MHCIl}^{\text {low-mid }}\right)$ in the BALF, as well as CD4 ${ }^{+}$cells in lung homogenate (h) were enumerated by flow cytometry. Each dot represents an individual mouse and is compiled from three independent experiments. Lines represent median values.
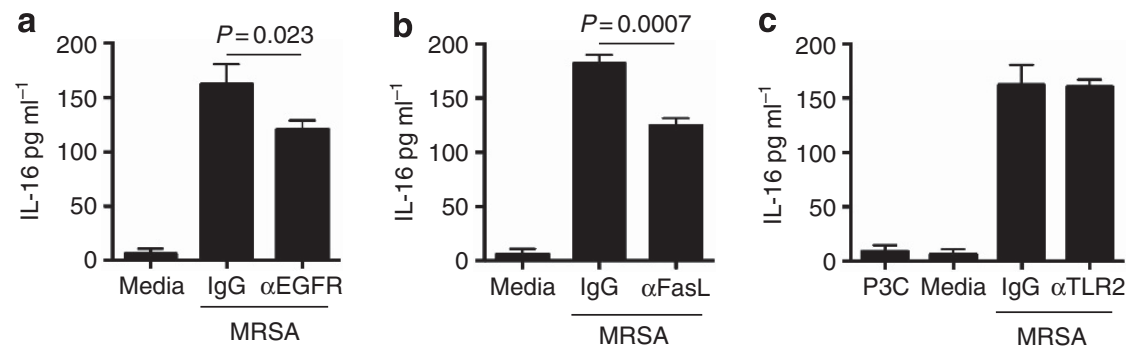

Figure 4 Multiple receptors are involved in methicillin-resistant S. aureus USA300 (MRSA) induced IL-16 secretion in THP-1 s. THP-1 s were pretreated with neutralizing antibody or immunoglobulin $\mathrm{G}(\mathrm{IgG})$ isotype $1 \mathrm{~h}$ before infection with MRSA at a multiplicity of infection of 50 . Neutralizing antibodies were (a) anti-epidermal growth factor receptor (EGFR) $(2 \mu \mathrm{g} / \mathrm{ml})$, (b) anti-Fas ligand $(5 \mu \mathrm{g} / \mathrm{ml})$ and $(\mathbf{c})$ anti-toll-like receptor $\left(\right.$ TLR2) $(2 \mu \mathrm{g} / \mathrm{ml})$. Pam ${ }_{3}$ Cys-Ser$\mathrm{Lys}_{4}$ (P3C) $(15 \mu \mathrm{g} / \mathrm{ml})$ was used as a control. $P$-values as compared with isotype controls, Student's $t$-test. The above graphs represent data from at least two independent experiments.

Supplementary Figure S1a,b online). THP-1s in media alone showed no baseline $\mathrm{Ca}^{2+}$ fluxes and gave significant fluorescence when treated with thapsigargin (Figure $\mathbf{5 b}$ and Supplementary Figure S1c). IL-16 secretion was stimulated by ionomycin alone and significantly decreased in the presence of EDTA $(P<0.05$ compared with media) (Figure 5c). Pretreatment of THP-1s with calpeptin, a calpain inhibitor, resulted in diminished secretion of IL-16 $(P<0.05$ compared with dimethyl sulfoxide (DMSO)) (Figure 5d), indicating that IL-16 processing in THP-1s is calpain dependent, as well as calcium dependent. Similar differences in Jurkats with calcium and calpain inhibitors were not seen (data not shown). Caspase 3 involvement was demonstrated when THP-1s preloaded with Caspase 3/7 Green Detection Reagent were infected with MRSA, showing an increase in fluorescence over baseline
(Figure 5e). We then observed decreased secretion of IL-16 when THP-1s were pretreated with pancaspase inhibitor Z-VAD-FMK $(P<0.05$ compared with DMSO with MRSA) or a caspase- 3 inhibitor $(P<0.05$ compared with DMSO with MRSA) but not a caspase-1 inhibitor (Figure 5f). Similarly, IL-16 release was reduced in Jurkats pretreated with caspase-3 inhibitor $(P<0.05$ compared with DMSO with MRSA) (Figure 5g). Staurosporine control, an in vitro inducer of caspase activity, ${ }^{30}$ showed induction of IL-16 secretion in both THP-1s and Jurkats.

\section{T cells are a major source of IL-16}

Our in vitro data suggest that multiple cells types can produce IL-16. To determine the major source of IL-16 in the setting of S. aureus pneumonia, immunohistochemistry was performed 
a

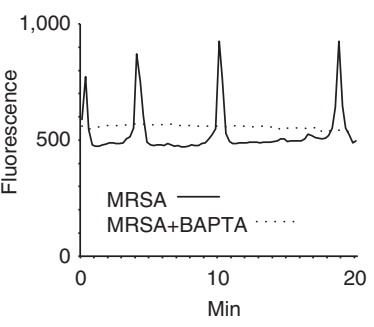

b

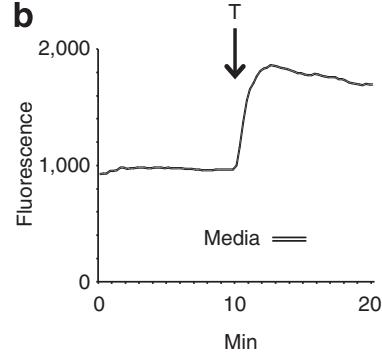

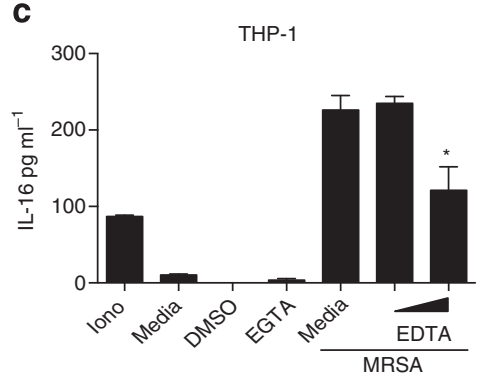

d

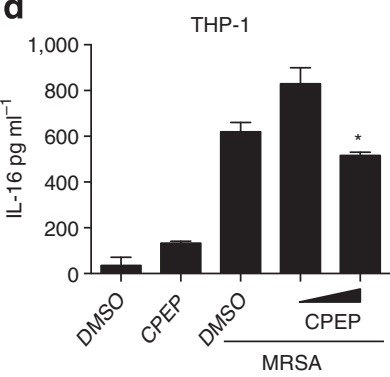

e

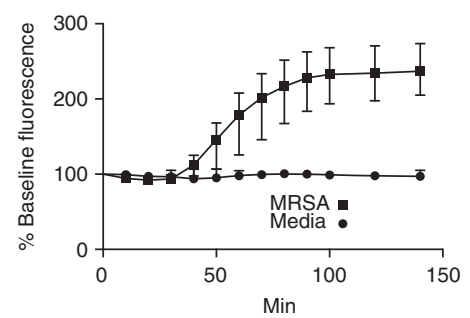

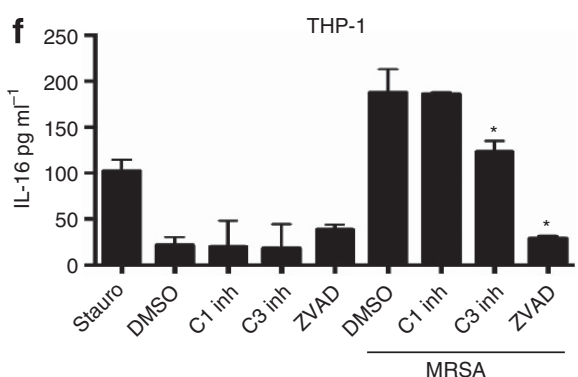

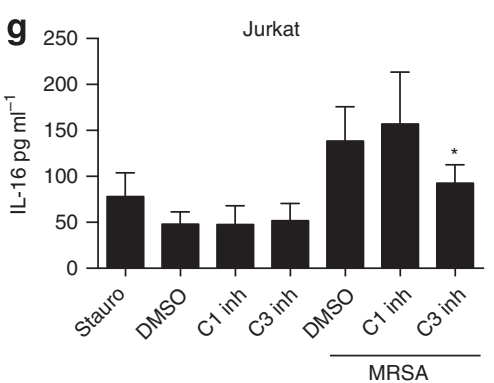

Figure 5 Methicillin-resistant S. aureus USA300 (MRSA) induction of interleukin-16 (IL-16) release is calcium and calpain dependent in THP-1s. Calcium fluxes were measured by fluorophore excitation with a mercury short arc lamp and imaged using an inverted microscope. THP-1s loaded with AM/Fluo-4 (1 mM) and PowerLoad concentrate $(1 \mathrm{~mm}) 2 \mathrm{~h}$ before stimulation with (a) MRSA multiplicity of infection (MOI) 100 with and without 1,2 bis (2-aminophenoxy)ethane-N,N,N9,N9-tetra-acetic acid (BAPTA) $(6 \mu \mathrm{m})$, or (b) media alone followed by thapsigargin $(1 \mu \mathrm{m})(\mathrm{T})$ as a positive control. Representative cells were chosen and fluorescence plotted over time. (c) IL-16 levels in supernatants of THP-1s pretreated $1 \mathrm{~h}$ before infection with EGTA ( 0.5 or $1 \mathrm{mmol}$ ) with MRSA (MOI 50) was measured $2 \mathrm{~h}$ after infection. (d) IL-16 levels in supernatants of THP-1s pretreated $1 \mathrm{~h}$ before infection with calpeptin (CPEP) (20 or $200 \mu \mathrm{m}$ ) with MRSA (MOI 50) was measured $2 \mathrm{~h}$ after infection. Ionomycin (iono) in DMSO was $5 \mathrm{mM}$. (e) THP-1s were preloaded with Caspase $3 / 7$ Green Detection agent $(5 \mu \mathrm{M}) 30$ min before infection and then infected with MRSA (MOI) 100). Fluorescence was then plotted over time. (f) IL-16 levels in supernatants of THP-1s pretreated $1 \mathrm{~h}$ before infection with respective inhibitor with MRSA (MOI 50) for $2 \mathrm{~h}$; caspase-1 inhibitor (C1 inh) $50 \mu \mathrm{m}$, caspase 3 inhibitor (C3 inh) $50 \mu \mathrm{m}$, and pancaspase inhibitor (ZVAD) $100 \mu \mathrm{m}$. Staurosporine in dimethyl sulfoxide (DMSO) was $1 \mu \mathrm{M}$. (g) IL-16 levels in supernatants of Jurkats pretreated $1 \mathrm{~h}$ before infection with respective inhibitor with MRSA (MOI 50) for $2 \mathrm{~h}$; caspase-1 inhibitor (C1 inh) $50 \mu \mathrm{m}$ and caspase 3 inhibitor (C3 inh) $50 \mu \mathrm{m}$. Staurosporine in DMSO was $1 \mu \mathrm{m} .{ }^{*} P<0.05$ value as compared with MRSA-infected samples, ANOVA, post hoc Dunnett's test for multiple comparisons. The above graphs represent data from at least two independent experiments.

on lung sections of WT mice infected with MRSA. IL-16 staining was observed in discrete cells (black arrows) throughout the lung tissue, and not confined to the respiratory epithelium in MRSA infection (Figure 6a). Since CD4 itself is a receptor for IL-16, we predicted that $\mathrm{CD}^{+}$cells would participate in an autocrine loop and were a likely source of the cytokine in the lung. $C d 4^{-1-}$ and WT mice were infected with MRSA, and at $24 \mathrm{~h}$ post inoculation, IL-16 in the BALF was nearly half in the airway of the $C d 4^{-/-}$mouse compared with WT counterparts $(P=0.0043)$ (Figure 6b). The apparent correlation between the abundance of $\mathrm{CD}^{+}$cells and IL-16 production was further confirmed in MRSA-infected mice with increased numbers of $\mathrm{CD}^{+}$cells. We noted that mice treated with clodronate, depleted of alveolar macrophages, had a significant increase in the number of pulmonary $\mathrm{CD} 4^{+}$cells (Figure $6 \mathrm{c}$ ), but not the other $\mathrm{T}$ cell populations. ${ }^{12}$ These mice had a corresponding increase in IL-16 in the setting of MRSA pneumonia (Figure 6d), suggesting $\mathrm{CD}^{+}$cells are stimulated by, as well as generated IL-16 in an autocrine manner.

\section{Neutralization of IL-16 improves clearance of MRSA and pathology}

The exuberant host response to infection is a hallmark of $S$. aureus pneumonia and we postulated that neutralization of
IL-16 would dampen its harmful consequences. By directly affecting $\mathrm{CD} 4{ }^{+}$cells, we hypothesized that we might limit the excessive proinflammatory response, without compromising the recruitment of phagocytes essential to clear the infection. As mice lacking IL-16 production are not available, we pretreated mice with anti-IL-16 before giving our standard intranasal inoculum of MRSA, documenting the expected reduction in the amount of IL-16 in the airway as compared with IgG control $(P=0.04)$ (Figure 7a). A major effect on MRSA clearance was observed with a sixfold reduction in colony-forming units recovered from the airways $(P=0.0042)$ (Figure $7 \mathbf{b})$ and better preservation of the normal lung architecture, than in the IgG-treated control (Figure 7c). Decreased amounts of KC/CXCL1 $(P=0.016)$ were seen in the neutralization group (Figure 7d), but the cellular populations recruited to the BALF and lung were unaffected (Supplementary Figure S2a-h). TNF, IL-6, and protein levels were similar (Figure 7e-g). Thus, IL-16 contributes to lung pathology in MRSA pneumonia.

\section{DISCUSSION}

IL-16 has been described in a number of inflammatory and oncologic conditions since its description by Cruikshank and co-workers. ${ }^{31}$ However, despite its importance in the development of $\mathrm{T}$ helper cell-mediated inflammation, ${ }^{32}$ relatively 
a

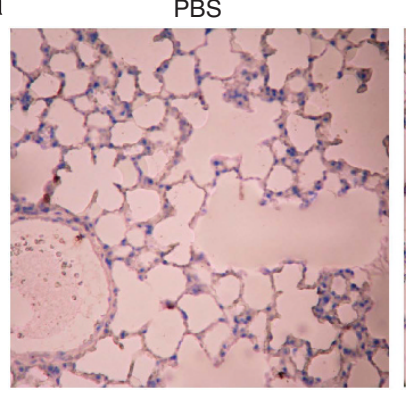

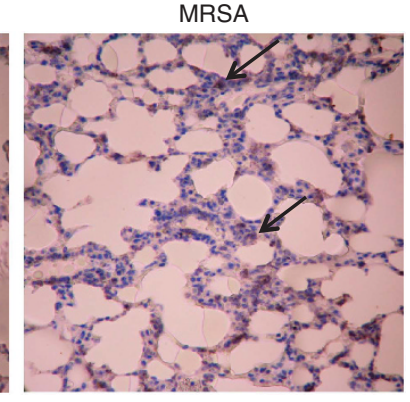
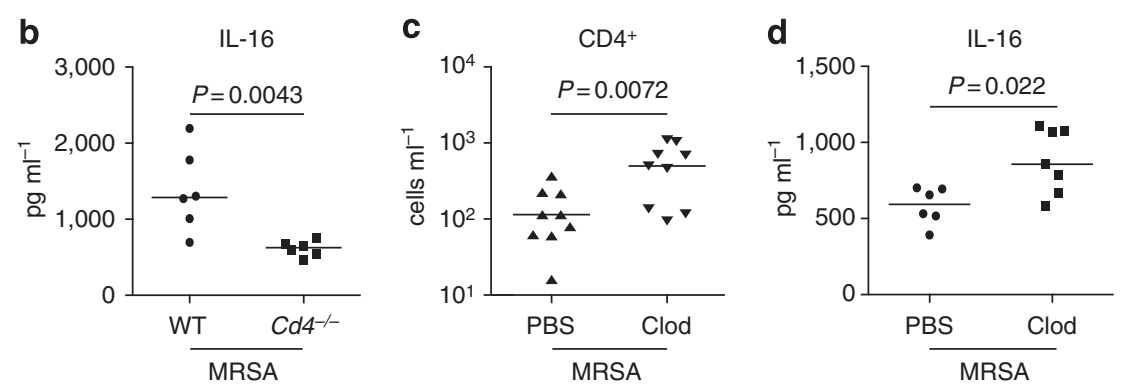

Figure 6 Interleukin-16 (IL-16) is produced by CD4 ${ }^{+}$cells. (a) Immunohistochemistry by standard methods with anti-IL-16 antibody on fixed-whole lungs of C57BL/6J WT given phosphate-buffered saline (PBS) or infected with $10^{7}$ colony-forming units methicillin-resistant S. aureus USA300 (MRSA) for $24 \mathrm{~h}$. Magnification $\times 100$. Black arrows point to areas of IL-16 staining (brown). (b) Age- and sex-matched cohorts of C57BL/6J wild type or Cd4 ${ }^{-/-}$ mice were inoculated intranasally with $10^{7}$ colony-forming units MRSA and IL-16 was quantified in bronchoalveolar lavage fluid (BALF) collected by ELISA. Clodronate (Clod) or PBS liposome-treated mice were given $10^{7}$ colony-forming units MRSA for $24 \mathrm{~h}$. BALF stained for (c) CD4 ${ }^{+}$populations were analyzed by flow cytometry and (d) IL-16 was measured by ELISA. Each dot represents an individual mouse and data is combined from two separate experiments. Lines represent median values. $P$-values are shown by Student's $t$-test.

little is known about its role in the pathogenesis of bacterial infection, including $S$. aureus pneumonia. ${ }^{33}$ The biology of murine and human IL-16 is highly conserved, ${ }^{34}$ thus the analysis of IL-16 in murine models of infection is likely applicable in human tissues as well. IL-16, as a ligand for CD4 ${ }^{+}$ cells, functions in $\mathrm{T}$ cell activation as well as proliferation ${ }^{35}$ and has biologic activity in both its proform and mature-processed form. ${ }^{36}$ We demonstrate that MRSA induces IL-16 acutely in the lung, where it participates in the exuberant and potentially damaging inflammatory response to infection.

Multiple cell types contribute to the proinflammatory signaling produced in response to $S$. aureus with the production of nuclear factor kappa-light-chain-enhancer of activated B cells (NF-kB)-dependent cytokines and chemokines. ${ }^{37}$ Epithelial, endothelial and immune cells can all produce IL-16 as part of the immediate response to MRSA. However, in contrast to the NF- $\kappa \mathrm{B}$ dependent cytokines, IL-16 production appears to be specifically linked to the TNF cascade and dependent upon both TNF and specific bacterial ligands. Lipopolysaccharide and TNF alone failed to stimulate IL-16 production, whereas MRSA and SpA alone were potent stimuli in most of the cell types examined. The central role of the TNF cascade in the induction of IL-16 processing, as demonstrated by diminished IL-16 production in infected mice lacking TNFR1, suggests an explanation for the enhanced ability of MRSA to activate this cytokine. In addition to stimulating TNF indirectly through production of PAMPs and pattern recognition receptors on immune cells, SpA directly ligates both TNFR1 and EGFR on multiple cell types to initiate signaling ${ }^{10,25}$ through redundant signaling pathways. IL-16 secretion into the airway of infected mice lacking TNFR1 was not fully attenuated and signaling by multiple receptors, including EGFR and Fas ligand, were found to initiate the processing of IL-16 to its active form.

A second stimulus, the generation of a $\mathrm{Ca}^{2+}$ flux sufficient to activate calpain activity was also required for IL-16 production. We demonstrated this response in THP-1s, representative of alveolar macrophages, the predominant immune cell to initially encounter respiratory pathogens. We were unable to show this calcium dependence in Jurkats, as $\mathrm{Ca}^{2+}$ fluxes are readily activated in T cells, ${ }^{38}$ which also possess the CD4 receptor for IL-16. In vivo, these $\mathrm{CD} 4^{+}$cells appear to be the most responsive to the multiple stimuli apparently required to initiate IL-16 processing. In vitro, we and others ${ }^{18}$ have demonstrated that multiple cell types can produce IL-16. However, the requirement for TNF signaling or ligation of a TNFR family receptor plus the need for calpain activity likely limits the in vivo production of the cytokine to specific cell types. This requirement for multiple stimuli may also help to explain why lipopolysaccharide alone, or the Gram-negative airway pathogens tested were much less capable in stimulating IL-16 production.

Once T cells are recruited, IL-16-CD4 interactions initiate an autocrine loop to further induce T cell production of more IL16. The ability of $S$. aureus to directly initiate a $\mathrm{T}$ cell cytokine response may contribute to virulence. The improved MRSA clearance in mice with decreased IL-16 suggests that $\mathrm{T}$ cell 

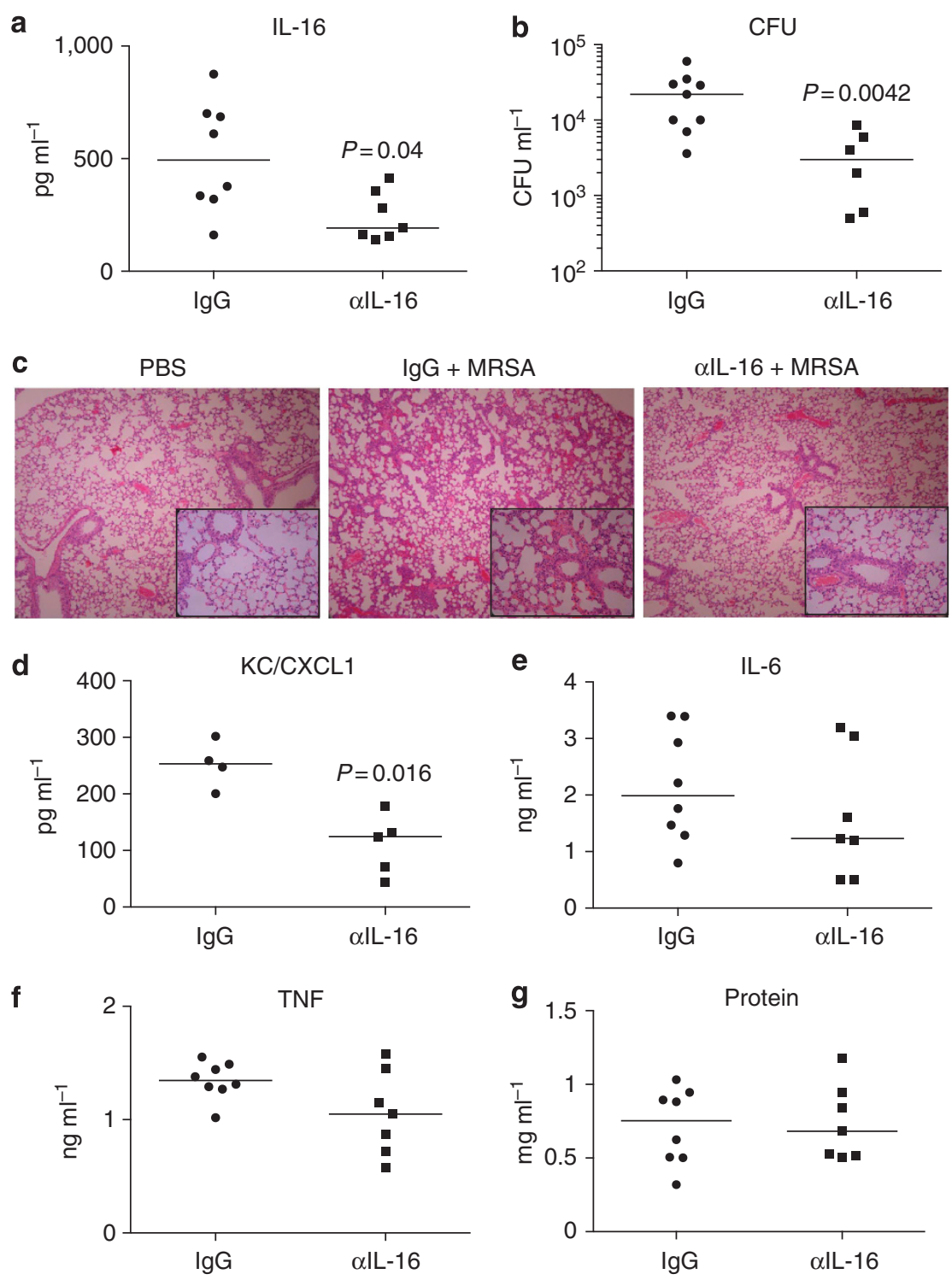

Figure 7 Neutralization of interleukin-16 (IL-16) improves clearance of methicillin-resistant S. aureus USA300 (MRSA). Anti-IL-16 antibody or immunoglobulin $\mathrm{G}(\mathrm{IgG})$ isotype control was injected i.p. $(100 \mu \mathrm{g}) 2 \mathrm{~h}$ before infection with $10^{7}$ colony-forming units (CFU) MRSA. Effect of IL-16 neutralization was documented by quantifying (a) IL-16 by ELISA and (b) bacterial clearance was measured by CFU in the bronchoalveolar lavage fluid (BALF) after $18 \mathrm{~h}$. (c) hematoxylin and eosin stain sections of fixed-whole lungs. Magnification $\times 100$, inset box $\times 400$. Cytokines (d) KC/CXCL1, (e) IL-6 and (f) tumor necrosis factor (TNF), as well as (g) protein were measured in the BALF. $P$-values are as shown, as compared with IgG isotype, by Student's $t$-test. Each dot represents an individual mouse and data is combined from two separate experiments. Lines represent median values.

recruitment and activation may contribute to lung damage in this setting. In our murine model system, we were unable to detect differences in the number of major immune cells recruited in mice treated with anti-IL-16 or the isotype control. Thus, the effects of the anti-IL-16 may be in blocking further $\mathrm{T}$ cell activity and their contribution to cytokine production.

In several other murine models of bacterial pneumonia, the ability to clear infection and preserve lung architecture correlated with decreased cytokine expression and not with the absolute numbers of inflammatory cells recruited into the lung. ${ }^{39,40}$ There are multiple potential sources of neutrophil and macrophage chemokines. ${ }^{37,41}$ Limiting the participation of
IL-16 and its T cell targets did not limit phagocytic influx, but resulted in decreased production of KC/CXCL1. Of note, $\mathrm{KC} / \mathrm{CXCL} 1$ is a major neutrophil chemokine that has been linked to lung injury in other models of sepsis ${ }^{42,43}$ consistent with the hypothesis that cytokines themselves, may be directly associated with lung damage.

In the absence of an $I l 16^{-/-}$mouse it is difficult to fully evaluate the role of this cytokine in the acute response to MRSA pneumonia. Our data indicate that the capability of MRSA, as opposed to commensal or opportunistic Gram-negative airway pathogens, to activate IL-16 and initiate the amplification of $\mathrm{CD} 4^{+} \mathrm{T}$ cell participation further contributes to its virulence in the setting of pneumonia. 


\section{METHODS}

Cell culture, bacterial strains, and purified proteins. Human airway epithelial cells (16HBEs, D. Gruenert, California Pacific Medical Center Research Institute, San Francisco, CA), HUVECs (B. Hopkins, Columbia College of Physicians and Surgeons, New York, NY), THP-1 human monocytic cells (THP-1s, ATCC, Manassas, VA), and Jurkat human T cells (Jurkats, E6-1, ATCC) were grown at $37^{\circ} \mathrm{C}$ with $5 \%$ $\mathrm{CO}_{2}$. THP-1s were activated with $1 \mu \mathrm{M}$ phorbol 12-myristate 13-acetate (PMA) and Jurkats were activated with $0.1 \mu \mathrm{M}$ PMA $+0.1 \mu \mathrm{M}$ ionomycin, both for $24 \mathrm{~h}$ before infection. Methicillin resistant $S$. aureus USA300 (MRSA), ${ }^{44}$ isogenic spa mutant, ${ }^{7}$ S. aureus 502A, K. pneumoniae ST258, and $P$. aeruginosa PAK were grown in Luria-Bertani broth and resuspended in antibiotic-free media 16HBEs-Bronchiolife (Lifeline Technologies, Frederick, MD) HUVECs-Vasculife (Lifeline Technologies) or THP-1s/JurkatsRPMI 1640 (Corning Cellgro, Manassas, VA), with 10\% heatinactivated fetal bovine serum, Benchmark (Gemini Bioproducts, West Sacremento, CA) for infections in vitro. Purified SpA (Calbiochem, Darmstadt, Germany) and TNF (Peprotech, Rocky Hill, NJ) were added at differential concentrations based on the assay. $\mathrm{Pam}_{3} \mathrm{Cys}-$ Ser-Lys 4 (EMC Microcollections, Tübingen, Germany), thapsigargin (Invitrogen, Grand Island, NY), ionomycin (Calbiochem), and staurosporine (Cell Signaling, Beverly, MA) were used as positive controls in respective assays.

Antibodies and Inhibitors. Antibodies used in vitro were anti-EGFR (1005; Santa Cruz, Dallas, TX), anti-Fas ligand (NOK1; BD Biosciences, San Jose, CA), anti-TLR2 (H-175; Santa Cruz), anti-TNFR1 (H-271; Santa Cruz), and anti-IL-16 (ab9563; Abcam, Cambridge, MA). Respective IgG isotype controls were used for comparison; rabbit (2027; Santa Cruz) or mouse (2025; Santa Cruz). Inhibitors used were pancaspase inhibitor Z-VAD-FMK (Santa Cruz), caspase 3 inhibitor (Calbiochem), caspase-1 inhibitor (Calbiochem), calpeptin (Calbiochem), BAPTA (Calbiochem) and EDTA (Sigma, St Louis, MO).

Mouse studies. In vivo experiments were performed using age- and sex-matched WT, Tnfr1 $1^{-/}$, and Cd4 ${ }^{-/-}$C57BL/6 J mice (Jackson Laboratories, Bar Harbor, ME). Mice were anaesthetized with $100 \mathrm{mg} /$ $\mathrm{kg}$ ketamine and $5 \mathrm{mg} / \mathrm{kg}$ xylazine, infected intranasally with MRSA, S. aureus 502A, K. pneumoniae ST258, or P. aeruginosa PAK $\left(10^{7}\right.$ colony-forming units in $50 \mu$ l of phosphate-buffered saline), and killed $18-24 \mathrm{~h}$ after infection. To deplete macrophages, $75 \mu \mathrm{l}$ of clodronate liposomes or phosphate-buffered saline liposome controls were inoculated intranasally $24 \mathrm{~h}$ before infection with MRSA as previously described. ${ }^{12}$ For the neutralization of IL-16, $100 \mu \mathrm{g}$ of anti-IL-16 antibody (ab9563; Abcam) or IgG isotype (Jackson ImmunoResearch, West Grove, PA) was injected intraperitoneally $2 \mathrm{~h}$ before infection as previously described. ${ }^{45}$ Animal experiments were performed in accordance with the guidelines of the IACUC at Columbia University (protocol number AAAE5252 and AAAD0624).

BALF Assays. BALF was obtained by instilling $1 \mathrm{ml}$ aliquots of sterile phosphate-buffered saline with calcium and magnesium into a cannulated trachea three times. Serial dilutions for bacterial enumeration were performed on the BALF before centrifuging. IL-16 mouse ELISA (R\&D Systems, Minneapolis, MN), TNF mouse ELISA (eBioscience, San Diego, CA), KC/CXCL1 mouse ELISA (R\&D systems), IL-6 mouse ELISA (Biolegend, San Diego, CA), and Bradford Assay (Thermo Scientific, Waltham, MA) for protein content were performed on the BALF supernatant.

Analysis of immune cell populations. Analysis of cell populations in BALF or single cell suspension of lung homogenate was conducted as before. ${ }^{46}$ Cells were labeled with a combination of phycoerythrin (PE)labeled anti-NK 1.1 (PK136; eBioscience), fluorescein isothiocyanate (FITC)-labeled anti-Ly6G (RB6-8C5; eBioscience), allophycocyanin (APC)-labeled anti-MHCII (M5/114.15.2; eBioscience), PerCP-Cy5.5labeled anti-CD11c (N418; Biolegend), or phycoerythrin (PE)-labeled
CD4 (RM4-5; Biolegend) alone. Polymorphonuclear neutrophils were defined as $\mathrm{Ly} 6 \mathrm{G}^{+} / \mathrm{MHCII}^{-}$, macrophages as $\mathrm{CD} 11 \mathrm{c}^{+} / \mathrm{MHCII}^{\text {low-mid }}$ and dendritic cells as $\mathrm{CD} 11 \mathrm{c}^{+} / \mathrm{MHCII}^{\text {high }}$. Fc block (anti-mouse CD16/32) was also added to each sample (93; eBioscience). Uniform-dyed microspheres (Bangs Laboratories, Fishers, IN) were added to calculate the concentration of cellular components. Samples were run on FACS Calibur (Becton Dickinson, Franklin Lakes, NJ) and analyzed on FlowJo (Tree Star, Ashland, OR).

Histopathology and Immunohistochemistry. Whole mouse lung was fixed with $4 \%$ paraformaldehyde for $24 \mathrm{~h}, 70 \%$ ethanol for $24 \mathrm{~h}$, and then prepared in paraffin blocks. Hematoxylin and eosin stain staining was performed on $5 \mathrm{~mm}$ sections for gross pathology. For immunohistochemistry, $5 \mathrm{~mm}$ sections on glass slides were rehydrated with xylene alternative (Safe Clear Protocol, Fischer Scientific, Pittsburg, PA) and ethanol. Antigens were presented using an acidic buffer (sodium citrate $10 \mathrm{~mm}, 0.05 \%$ Tween $20, \mathrm{pH} 6$ ) at $60^{\circ} \mathrm{C}$ overnight. Sections were additionally permeabilized with Tween $200.5 \%$ for $10 \mathrm{~min}$ and quenched with $0.3 \% \mathrm{H}_{2} \mathrm{O}_{2}$ for 10 min. Detection of IL-16 by immunohistochemistry with peroxidase staining was performed using the Immunocruz ABC Staining kit (Santa Cruz) using anti-IL-16 antibody (ab9563, Abcam). Controls were performed with secondary antibody only.

Calcium Imaging. THP-1s were loaded with acetoxymethyl ester fluorescent dyes/Fluo-4 (Invitrogen) with PowerLoad concentrate (Invitrogen) before imaging and stimulation. Fluorophores were excited with a 103W mercury short arc HBO lamp (Osram, Munich, Germany) with the illumination directed through appropriate interference filters. Fluorophore exposures were controlled by a filter wheel (LAMBDA10-2; Sutter Instrument, Novato, CA). Cells were then imaged on an Olympus IX81 Inverted microscope (Central Valley, PA). Images were analyzed and videos made using Metamorph software (Ver. 7.5.3.0, April, 2008), Molecular Devices, Dowington, PA).

Caspase 3/7-detection Assay. THP-1s were preloaded with CellEvent Caspase 3/7 Green Detection Reagent (Invitrogen) $30 \mathrm{~min}$ before infection. Cells were stimulated and then read at absorption/emission maxima of $502 / 530 \mathrm{~nm}$ every $10 \mathrm{~min}$ for $2 \mathrm{~h}$ on a Tecan Infinite 200 (V2.11, April, 2008, Tecan Infinite 200, Seestrasse, Germany).

Statistics. All statistical analysis was performed using GraphPad Prism Version 6.0c (21 March 2013, La Jolla, CA). Samples with normal distributions, were analyzed by Student's $t$-test. Mouse samples were compared using the nonparametric Mann-Whitney test. For multiple comparisons, an analysis of variance was then followed by a post hoc Dunnett's test to compare with the control group. Differences in groups were considered significant if $P<0.05$.

SUPPLEMENTARY MATERIAL is linked to the online version of the paper at http://www.nature.com/mi

\section{ACKNOWLEDGMENTS}

This work was funded by R01HL079395 to AP. PJP is supported by K08Al101005. Fluorescence microscopy was performed in the laboratory of Jahar Bhattacharya with the gracious assistance of Kristen Westphalen at the Columbia University College of Physicians and Surgeons, New York, NY, USA.

\section{DISCLOSURE}

The authors declare no conflict of interest.

(c) 2014 Society for Mucosal Immunology

\section{REFERENCES}

1. Klevens, R.M. et al. Invasive methicillin-resistant Staphylococcus aureus infections in the United States. JAMA 298, 1763-1771 (2007). 
2. American Thoracic Society; Infectious Diseases Society of America. Guidelines for the management of adults with hospital-acquired, ventilatorassociated, and healthcare-associated pneumonia. Am. J. Respir. Crit. Care Med 171, 388-416 (2005).

3. Morens, D.M., Taubenberger, J.K. \& Fauci, A.S. Predominant role of bacterial pneumonia as a cause of death in pandemic influenza: implications for pandemic influenza preparedness. J. Infect. Dis. 198, 962-970 (2008).

4. Kollef, M.H. et al. Epidemiology and outcomes of health-care-associated pneumonia results from a large us database of culture-positive pneumonia. Chest 128, 3854-3862 (2005).

5. Inoshima, I. et al. A Staphylococcus aureus pore-forming toxin subverts the activity of ADAM10 to cause lethal infection in mice. Nat. Med. 17, 1310-1314 (2011).

6. Diep, B.A. et al. Polymorphonuclear leukocytes mediate Staphylococcus aureus Panton-Valentine leukocidin-induced lung inflammation and injury. Proc. Natl. Acad. Sci. USA. 107, 5587-5592 (2010).

7. Martin, F.J. et al. Staphylococcus aureus activates type I IFN signaling in mice and humans through the Xr repeated sequences of protein A. J. Clin. Invest. 119, 1931-1939 (2009)

8. Kebaier, C. et al. Staphylococcus aureus $\alpha$-hemolysin mediates virulence in a murine model of severe pneumonia through activation of the NLRP3 inflammasome. J. Infect. Dis. 205, 807-817 (2012).

9. Gomez, M.I. et al. Staphylococcus aureus protein A induces airway epithelial inflammatory responses by activating TNFR1. Nat. Med. 10, 842-848 (2004).

10. Gomez, M.I. Staphylococcus aureus protein A activates TNFR1 signaling through conserved IgG binding domains. J. Biol. Chem. 281, 20190-20196 (2006).

11. Ichikawa, A. et al. CXCL10-CXCR3 enhances the development of neutrophil-mediated fulminant lung injury of viral and nonviral origin. Am. J. Respir. Crit. Care Med. 187, 65-77 (2013).

12. Martin, F.J., Parker, D., Harfenist, B.S., Soong, G. \& Prince, A. Participation of $\mathrm{CD} 11 \mathrm{c}(+)$ leukocytes in methicillin-resistant Staphylococcus aureus clearance from the lung. Infect. Immun. 79, 1898-1904 (2011).

13. McLoughlin, R.M. et al. CD4 + Tcells and CXC chemokines modulate the pathogenesis of Staphylococcus aureus wound infections. Proc. Natl. Acad. Sci. USA. 103, 10408-10413 (2006).

14. Köckritz-Blickwede, von, M. et al. Immunological mechanisms underlying the genetic predisposition to severe staphylococcus aureus infection in the mouse model. Am. J. Pathol. 173, 1657-1668 (2008).

15. Wilson, G.J. et al. A novel core genome-encoded superantigen contributes to lethality of community-associated MRSA necrotizing pneumonia. PLoS Pathog. 7, e1002271 (2011).

16. Jiang, D. et al. Long-term exposure of chemokine CXCL10 causes bronchiolitis-like inflammation. Am. J. Respir. Cell Mol. Biol. 46, 592-598 (2012).

17. Hamza, T., Barnett, J.B. \& Li, B. Interleukin 12 a key immunoregulatory cytokine in infection applications. Int. J. Mol. Sci. 11, 789-806 (2010).

18. Cruikshank, W.W., Kornfeld, H. \& Center, D.M. Interleukin-16. J. Leukoc. Biol. 67, 757-766 (2000).

19. Cruikshank, W. \& Center, D.M. Modulation of lymphocyte migration by human lymphokines. II. Purification of a lymphotactic factor (LCF). J. Immunol. 128, 2569-2574 (1982).

20. Cruikshank, W. \& Little, F. Interleukin-16: the ins and outs of regulating Tcell activation. Crit. Rev. Immunol. 28, 467-483 (2008).

21. Little, F.F., Lynch, E., Fine, G., Center, D.M. \& Cruikshank, W.W. Tumor necrosis factor- $\alpha$-induced synthesis of interleukin-16 in airway epithelial cells. Am. J. Respir. Cell Mol. Biol. 28, 354-362 (2003).

22. Zhang, Y. et al. Processing and activation of pro-interleukin- 16 by caspase3. J. Biol. Chem. 273, 1144-1149 (1998).

23. Aggarwal, B.B. Signalling pathways of the TNF superfamily: a doubleedged sword. Nat. Rev. Immunol. 3, 745-756 (2003).
24. Wang, P. et al. IL-16 Induces intestinal inflammation via PepT1 upregulation in a pufferfish model: new insights into the molecular mechanism of inflammatory bowel disease. J. Immunol. 191, 1413-1427 (2013).

25. Gomez, M.I., Seaghdha, M.O. \& Prince, A.S. Staphylococcus aureus protein A activates TACE through EGFR-dependent signaling. EMBO J. 26, 701-709 (2007).

26. Lavrik, I.N. \& Krammer, P.H. Regulation of CD95/Fas signaling at the DISC. Cell Death Differ. 19, 36-41 (2011).

27. Vaisid, T., Barnoy, S. \& Kosower, N.S. Calpain activates caspase-8 in neuron-like differentiated PC12 cells via the amyloid-beta-peptide and CD95 pathways. Int. J. Biochem. Cell Biol. 41, 2450-2458 (2009).

28. Chun, J. \& Prince, A. Activation of $\mathrm{Ca} 2+$-dependent signaling by TLR2. J. Immunol. 177, 1330-1337 (2006).

29. Takeuchi, O., Hoshino, K. \& Akira, S. Cutting edge: TLR2-deficient and MyD88-deficient mice are highly susceptible to Staphylococcus aureus infection. J. Immunol. 165, 5392-5396 (2000).

30. Chae, H. Molecular mechanism of staurosporine-induced apoptosis in osteoblasts. Pharmacol. Res. 42, 373-381 (2000).

31. Center, D.M. \& Cruikshank, W. Modulation of lymphocyte migration by human lymphokines. I. Identification and characterization of chemoattractant activity for lymphocytes from mitogen-stimulated mononuclear cells. J. Immunol. 128, 2563-2568 (1982).

32. Lynch, E.A., Heijens, C.A.W., Horst, N.F., Center, D.M. \& Cruikshank, W.W. Cutting edge: IL-16/CD4 preferentially induces Th1 cell migration: requirement of CCR5. J. Immunol. 171, 4965-4968 (2003).

33. Glass, W.G., Sarisky, R.T. \& Vecchio, A.M.D. Not-so-sweet sixteen: the role of il-16 in infectious and immune-mediated inflammatory diseases. J. Interferon Cytokine Res. 26, 511-520 (2006).

34. Keane, J. et al. Conservation of structure and function between human and murine IL-16. J. Immunol. 160, 5945-5954 (1998).

35. Wilson, K.C., Center, D.M. \& Cruikshank, W.W. The effect of interleukin-16 and its precursor on T lymphocyte activation and growth. Growth Factors 22, 97-104 (2004).

36. Ren, F. et al. Pro-IL-16 regulation in activated murine CD4 + lymphocytes. J. Immunol. 174, 2738-2745 (2005).

37. Parker, D. \& Prince, A. Immunopathogenesis of Staphylococcus aureus pulmonary infection. Semin. Immunopathol. 34, 281-297 (2012).

38. Hogan, P.G., Lewis, R.S. \& Rao, A. Molecular basis of calcium signaling in Iymphocytes: STIM and ORAI. Annu. Rev. Immunol. 28, 491-533 (2010).

39. Cohen, T.S. \& Prince, A.S. Bacterial pathogens activate a common inflammatory pathway through IFNג regulation of PDCD4. PLoS Pathog. 9, e1003682 (2013).

40. Cohen, T.S. \& Prince, A.S. Activation of inflammasome signaling mediates pathology of acute P. aeruginosa pneumonia. J. Clin. Invest. 123, 1630-1637 (2013).

41. Archer, N.K., Harro, J.M. \& Shirtliff, M.E. Clearance of Staphylococcus aureus nasal carriage is t cell dependent and mediated through interleukin17a expression and neutrophil influx. Infect. Immun. 81, 2070-2075 (2013).

42. Ahuja, N. et al. Circulating IL-6 mediates lung injury via CXCL1 production after acute kidney injury in mice. Am. J. Physiol. Renal Physiol. 303, F864-F872 (2012)

43. Neumann, B. et al. Mechanisms of acute inflammatory lung injury induced by abdominal sepsis. Int. Immunol. 11, 217-227 (1999).

44. Diep, B.A. et al. Complete genome sequence of USA300, an epidemic clone of community-acquired meticillin-resistant Staphylococcus aureus. Lancet 367, 731-739 (2006).

45. Meagher, C. et al. Neutralization of interleukin-16 protects nonobese diabetic mice from autoimmune type 1 diabetes by a CCL4-dependent mechanism. Diabetes 59, 2862-2871 (2010).

46. Parker, D. et al. Streptococcus pneumoniae DNA initiates type I interferon signaling in the respiratory tract. MBio. 2, e00016-11 (2011). 